\title{
Positive solutions for Sturm-Liouville BVPs on time scales via sub-supersolution and variational methods
}

\author{
Quan-Guo Zhang ${ }^{1}$, Xi-Ping He $\mathrm{H}^{2}$ and Hong-Rui Sun ${ }^{1 *}$
}

*Correspondence: hrsun@lzu.edu.cn
${ }^{1}$ School of Mathematics and
Statistics, Lanzhou University,
Lanzhou, Gansu 730000, People's
Republic of China
Full list of author information is
available at the end of the article

*Correspondence: hrsun@lzu.edu.cn 'School of Mathematics and Republic of China

available at the end of the article

\begin{abstract}
This paper is concerned with the existence of one and two positive solutions for the following Sturm-Liouville boundary value problem on time scales

$$
\left\{\begin{array}{l}
-\left(p(t) u^{\Delta}(t)\right)^{\Delta}+q(t) u^{\sigma}(t)=f\left(t, u^{\sigma}(t)\right), \quad t \in[0, T]_{\mathbb{T}} \\
\alpha_{1} u(0)-\alpha_{2} u^{\Delta}(0)=0, \quad \alpha_{3} u\left(\sigma^{2}(T)\right)+\alpha_{4} u^{\Delta}(\sigma(T))=0 .
\end{array}\right.
$$

Under a locally nonnegative assumption on the nonlinearity $f$ and some other suitable hypotheses, positive solutions are sought by considering the corresponding truncated problem, constructing the variational framework and combining the sub-supersolution method with the mountain pass lemma.

MSC: $34 \mathrm{~B} 10 ; 34 \mathrm{~B} 18$
\end{abstract}

Keywords: positive solution; Sturm-Liouville; time scales; sub-supersolution; variational methods

\section{Introduction}

The theory of dynamic equations on time scales has become a new important mathematical branch [1, 2] since it was initiated by Hilger in 1988 [3]. Since then, boundary value problems (BVPs) for dynamic equations on time scales have received considerable attention, various fixed point theorems, sub-supersolution method and Leray-Schauder degree theory have been applied to get many interesting results about the existence of solutions; see $[1,2,4-12]$ and the references therein. Variational method is also an important method for dealing with the existence of BVPs. Recently, some authors have used the theory to study the existence of solutions of some BVPs on time scales $[4,5,13,14]$.

Especially, in [4, 5], Agarwal et al. studied the following dynamic equation on time scales:

$$
-u^{\Delta \Delta}(t)=f\left(t, u^{\sigma}(t)\right), \quad t \in(a, b)_{\mathbb{T}},
$$

and

$$
-u^{\Delta \Delta}(t)=f\left(\sigma(t), u^{\sigma}(t)\right), \quad t \in(a, b)_{\mathbb{T}},
$$


with the Dirichlet boundary condition. They gave some sufficient conditions for the existence of single and multiple positive solutions by using the variational method and critical point theory.

In [14], we considered the problem

$$
\left\{\begin{array}{l}
-\left(p(t) u^{\Delta}(t)\right)^{\Delta}+q(t) u^{\sigma}(t)=\lambda f\left(t, u^{\sigma}(t)\right), \quad t \in[0, T]_{\mathbb{T}}, \\
\alpha_{1} u(0)-\alpha_{2} u^{\Delta}(0)=0, \quad \alpha_{3} u\left(\sigma^{2}(T)\right)+\alpha_{4} u^{\Delta}(\sigma(T))=0,
\end{array}\right.
$$

and obtained the existence of many solutions depending on the value of the parameter $\lambda$ which lie in some different intervals under some suitable hypotheses. The main approaches are also the variational method and some known critical point theorems and a three critical point theorem established in [15]. Erbe et al. [8] also established some existence criteria of positive solutions by a fixed point theorem in a cone with the globally nonnegative hypothesis of $f$.

Motivated by the papers $[4,5,8,14]$, in this paper, we continue to study the problem (1.1) in the case of $\lambda=1$, that is,

$$
\left\{\begin{array}{l}
-\left(p(t) u^{\Delta}(t)\right)^{\Delta}+q(t) u^{\sigma}(t)=f\left(t, u^{\sigma}(t)\right), \quad t \in[0, T]_{\mathbb{T}}, \\
\alpha_{1} u(0)-\alpha_{2} u^{\Delta}(0)=0, \quad \alpha_{3} u\left(\sigma^{2}(T)\right)+\alpha_{4} u^{\Delta}(\sigma(T))=0 .
\end{array}\right.
$$

Here $\mathbb{T}$ is a time scale and $[0, T]_{\mathbb{T}}=\left\{t \in \mathbb{T}: 0 \leq t \leq T\right.$ and $\left.T \in \mathbb{T}^{\kappa^{2}}\right\}, p \in C^{1}\left([0, \sigma(T)]_{\mathbb{T}}\right.$, $(0,+\infty)), q \in C\left([0, T]_{\mathbb{T}},[0,+\infty)\right), f \in C\left([0, T]_{\mathbb{T}} \times \mathbb{R}, \mathbb{R}\right), \alpha_{i} \geq 0, i=1,2,3,4, \alpha_{1}+\alpha_{2}>0, \alpha_{3}+$ $\alpha_{4}>0$ and $\alpha_{1}+\alpha_{3}>0$. The purpose of this paper is to discuss the existence and multiplicity of positive solutions to the problem (1.2) under the local non-negativity assumption of $f$ and some other hypotheses. The main tools are the truncated method, the variational method, the sub-supersolution method and the mountain pass lemma. First, inspired by the method in [4], we convert the existence of a positive solution of (1.2) to the existence of a solution of an associated problem of (1.2). In contrast with the paper [4], the appearance of term $p(t)$, our problem is more complicated and the proof is also different from [4] (see Lemma 2.3 for details). Next, we construct a supersolution of (1.2) and give the existence of one positive solution. Finally, under our weaker assumption on $f$ (see (H1) and (H2) for details), since we cannot verify that the corresponding functional for the associated problem satisfies the P.S. condition, we consider the corresponding truncated problem. To prove the existence of the second positive solution by the mountain pass lemma, we also give an estimate of a nonnegative solution of (1.2) and prove the solution of a truncated problem is also a solution of (1.2) for $n$ large enough (see Theorem 3.3 for details). To the best of our knowledge, the results are new both in the continuous and in the discrete case.

The paper is organized as follows. In Section 2, we present some basic properties of some related Sobolev space on time scales, construct the variational framework, give some properties of this framework and some necessary lemmas. In Section 3, we firstly get the existence of a single positive solution of (1.2) by using the sub-supersolution method; then applying the truncated method, analytic technique and mountain pass lemma, we establish the existence of two positive solutions. 


\section{Preliminaries and variational formulation}

In this section, we list the definition and properties of the Sobolev space on time scales [16], give some lemmas which we need for the proof of the main result and construct a variational framework.

For convenience, for $f \in L_{\Delta}^{1}\left([a, b)_{\mathbb{T}}\right)[16,17]$, we denote $\int_{a}^{b} f(s) \Delta s=\int_{[a, b)} f(s) \Delta s$. We let $A C\left(\left[0, \sigma^{2}(T)\right]_{\mathbb{T}}\right)[18]$ denote the class of absolutely continuous functions on $\left[0, \sigma^{2}(T)\right]_{\mathbb{T}}$ and the Sobolev space is defined as

$$
H_{\Delta}^{1}\left(\left[0, \sigma^{2}(T)\right]_{\mathbb{T}}\right)=\left\{u \mid u \in A C\left[0, \sigma^{2}(T)\right]_{\mathbb{T}}, u^{\Delta} \in L_{\Delta}^{2}\left(\left[0, \sigma^{2}(T)\right)_{\mathbb{T}}\right)\right\},
$$

with the norm

$$
\|u\|=\left(\int_{0}^{\sigma^{2}(T)}\left(|u(t)|^{2}+\left|u^{\Delta}(t)\right|^{2}\right) \Delta t\right)^{\frac{1}{2}}
$$

We know the immersion $H_{\Delta}^{1}\left(\left[0, \sigma^{2}(T)\right]_{\mathbb{T}}\right) \hookrightarrow C\left(\left[0, \sigma^{2}(T)\right]_{\mathbb{T}}\right)$ is compact. Analogous to the proof in the real numbers situation, one can deduce the following result on time scales.

Lemma 2.1 If $f \in C^{1}(\mathbb{R}), f^{\prime} \in L^{\infty}(\mathbb{R}), u \in H_{\Delta}^{1}\left(\left[0, \sigma^{2}(T)\right]_{\mathbb{T}}\right)$, then

$$
f(u) \in H_{\Delta}^{1}\left(\left[0, \sigma^{2}(T)\right]_{\mathbb{T}}\right)
$$

and

$$
(f(u(t)))^{\Delta}=u^{\Delta}(t) \int_{0}^{1} f^{\prime}(u(t)+(\sigma(t)-t) s) d s
$$

Using Lemma 2.1, by a similar proof of $\mathbb{T}=\mathbb{R}$, one can derive the following.

Lemma 2.2 If $u \in H_{\Delta}^{1}\left(\left[0, \sigma^{2}(T)\right]_{\mathbb{T}}\right)$, then $u^{+}, u^{-} \in H_{\Delta}^{1}\left(\left[0, \sigma^{2}(T)\right]_{\mathbb{T}}\right)$ and $\left(u^{+}\right)^{\Delta}\left(u^{-}\right)^{\Delta} \geq 0$, $\Delta$-a.e. in $\left[0, \sigma^{2}(T)\right]_{\mathbb{T}}$, where $u^{+}=\max \{u, 0\}, u^{-}=-(-u)^{+}$.

For convenience, we denote

$$
\beta_{1}=\left\{\begin{array}{ll}
\frac{\alpha_{1}}{\alpha_{2}} & \text { if } \alpha_{2} \neq 0, \\
0 & \text { if } \alpha_{2}=0,
\end{array} \quad \beta_{2}= \begin{cases}\frac{\alpha_{3}}{\alpha_{4}} & \text { if } \alpha_{4} \neq 0 \\
0 & \text { if } \alpha_{4}=0\end{cases}\right.
$$

and for $u, v \in H_{\Delta}^{1}\left(\left[0, \sigma^{2}(T)\right]_{\mathbb{T}}\right)$, we set

$$
\begin{aligned}
(u, v)_{0}= & \int_{0}^{\sigma^{2}(T)} p(t) u^{\Delta}(t) v^{\Delta}(t) \Delta t+\int_{0}^{\sigma(T)} q(t) u^{\sigma}(t) v^{\sigma}(t) \Delta t+\beta_{1} p(0) u(0) v(0) \\
& +\beta_{2} p(\sigma(T)) u\left(\sigma^{2}(T)\right) v\left(\sigma^{2}(T)\right), \\
\|u\|_{0}= & \sqrt{(u, u)_{0}} .
\end{aligned}
$$


In order to discuss the existence of a positive solution of (1.2), we consider the following problem:

$$
\left\{\begin{array}{l}
-\left(p(t) u^{\Delta}(t)\right)^{\Delta}+q(t) u^{\sigma}(t)=f\left(t,\left(u^{+}\right)^{\sigma}(t)\right), \quad t \in[0, T]_{\mathbb{T}}, \\
\alpha_{1} u(0)-\alpha_{2} u^{\Delta}(0)=0, \quad \alpha_{3} u\left(\sigma^{2}(T)\right)+\alpha_{4} u^{\Delta}(\sigma(T))=0 .
\end{array}\right.
$$

First, we give an important lemma.

Lemma 2.3 If $f(t, 0) \geq 0$ for $t \in[0, T]_{\mathbb{T}}, u$ is a solution of (2.1), then $u$ is nonnegative in $\left[0, \sigma^{2}(T)\right]_{\mathbb{T}}$. Furthermore, if $f(t, 0)>0$ for $t \in[0, T]_{\mathbb{T}}$, and $p(t)$ is nondecreasing in $[0, \sigma(T)]_{\mathbb{T}}$, then $u(t)>0, t \in\left(0, \sigma^{2}(T)\right)_{\mathbb{T}}$.

Proof Let $u$ be a solution of (2.1). In view of Lemma 2.2, we know $u^{+}, u^{-} \in H_{\Delta}^{1}\left(\left[0, \sigma^{2}(T)\right]_{\mathbb{T}}\right)$ and $\left(u^{+}\right)^{\Delta}\left(u^{-}\right)^{\Delta} \geq 0, \Delta$-a.e. in $\left[0, \sigma^{2}(T)\right]_{\mathbb{T}}$. Multiplying $(2.1)$ by $\left(u^{-}\right)^{\sigma}(t)$, integrating over $[0, \sigma(T))_{\mathbb{T}}$ and employing the integration by parts formula for an absolutely continuous function on $\mathbb{T}$, we find that

$$
\begin{aligned}
\left\|u^{-}\right\|_{0}^{2} \leq & \int_{0}^{\sigma^{2}(T)} p(t) u^{\Delta}(t)\left(u^{-}\right)^{\Delta}(t) \Delta t+\int_{0}^{\sigma(T)} q(t) u^{\sigma}(t)\left(u^{-}\right)^{\sigma}(t) \Delta t \\
& +\beta_{1} p(0) u(0) u^{-}(0)+\beta_{2} p(\sigma(T)) u\left(\sigma^{2}(T)\right) u^{-}\left(\sigma^{2}(T)\right) \\
= & \int_{0}^{\sigma(T)} f\left(t,\left(u^{+}\right)^{\sigma}(t)\right)\left(u^{-}\right)^{\sigma}(t) \Delta t \\
= & \int_{0}^{\sigma(T)} f(t, 0)\left(u^{-}\right)^{\sigma}(t) \Delta t \leq 0 .
\end{aligned}
$$

Therefore, $u(t) \geq 0$ for $t \in\left[0, \sigma^{2}(T)\right]_{\mathbb{T}}$.

Next, we show that if $p(t)$ is nondecreasing in $[0, \sigma(T)]_{\mathbb{T}}$, and $f(t, 0)>0$ for $t \in[0, T]_{\mathbb{T}}$, then $u(t)>0, t \in\left(0, \sigma^{2}(T)\right)_{\mathbb{T}}$.

In fact, if the conclusion is false, we can suppose that there exists $c \in\left(0, \sigma^{2}(T)\right)_{\mathbb{T}}$ such that $u(c)=0$ and one of the following two cases holds:

(i) $u(t)>0$ for $t \in\left(c, \sigma^{2}(T)\right)_{\mathbb{T}}$ and $\sigma(c) \neq \sigma^{2}(T)$,

(ii) $u(t)=0$ for $t \in\left(c, \sigma^{2}(T)\right)_{\mathbb{T}}$ and there exists $\delta>0$ such that $u(t)>0$ for $t \in[c-\delta, c)_{\mathbb{T}}$. For the case (i), if $\rho(c)=c<\sigma(c)$, then $u^{\Delta}(c)>0$, there exists $\delta>0$ such that $u^{\Delta}(t)>0$ for $t \in(c-\delta, c]_{\mathbb{T}}$. According to the nonnegativity of $u$ on $\left[0, \sigma^{2}(T)\right]_{\mathbb{T}}$ and $u(c)=0$, it is easy to see that $\rho(c)=c<\sigma(c)$ is impossible. Thus we have

$$
\sigma(\rho(c))=c
$$

If $\rho(c)<c \leq \sigma(c)$, then we know $u^{\Delta}(c) \geq 0, u^{\Delta}(\rho(c)) \leq 0$ and $u^{\Delta \Delta}(\rho(c))=\frac{u^{\Delta}(c)-u^{\Delta}(\rho(c))}{c-\rho(c)} \geq 0$. If $\rho(c)=c=\sigma(c)$, then $u^{\Delta}(c)=0, u^{\Delta \Delta}(\rho(c)) \geq 0$. Hence, in this case, we always have

$$
u^{\Delta}(c) \geq 0, \quad u^{\Delta \Delta}(\rho(c)) \geq 0 .
$$

Therefore

$$
-\left(p(\rho(c)) u^{\Delta}(\rho(c))\right)^{\Delta}=-p(\rho(c)) u^{\Delta \Delta}(\rho(c))-p^{\Delta}(\rho(c)) u^{\Delta}(c) \leq 0 .
$$


However, since $u$ is a solution of (1.2), (2.2) and $u(c)=0$, we know that

$$
-\left(p(\rho(c)) u^{\Delta}(\rho(c))\right)^{\Delta}=f(\rho(c), 0)>0,
$$

which contradicts (2.4).

For the case (ii), it can be divided into two cases to consider.

(1) If $\sigma(c)<\sigma^{2}(T)$, then one can deduce that $u^{\Delta}(c)=0, u^{\Delta \Delta}(c) \geq 0$. From this and $-p(\sigma(c)) u^{\Delta \Delta}(c)=f(c, 0)>0$, we have a contradiction.

(2) If $\sigma(c)=\sigma^{2}(T)$, then we always have $\sigma(c)>c$. If $u\left(\sigma^{2}(T)\right)>0$, then $u^{\Delta}(c)>0$, $u^{\Delta \Delta}(\rho(c)) \geq 0$. Similar to (i), we get (2.2) and (2.3). But this is impossible from (2.4) and (2.5).

If $u\left(\sigma^{2}(T)\right)=0$ and $c=\sigma(T)$, then $\sigma(T)>T, u^{\Delta}(c)=u^{\Delta}(\sigma(T))=0, u^{\Delta \Delta}(\rho(c))=$ $u^{\Delta \Delta}(T) \geq 0$. So, we get a contradiction to (2.4) and (2.5).

If $u\left(\sigma^{2}(T)\right)=0$ and $\rho(c)<c<\sigma(T)$, we have $u^{\Delta}(c)=0, u^{\Delta \Delta}(\rho(c)) \geq 0$. Hence, we get (2.2) and (2.3). But this contradicts (2.4) and (2.5).

If $u\left(\sigma^{2}(T)\right)=0$ and $\rho(c)=c<\sigma(T)$, then $u^{\Delta}(c)=0$. By assumption, $u$ is a solution of (1.2), so we have $\lim _{t \rightarrow c^{-}} u^{\Delta \Delta}(t)<0$, which contradicts $u^{\Delta}(c)=0$ and $u(t)>0$ for $t \in[c-\delta, c)_{\mathbb{T}}$.

Remark 2.4 From the proof of Lemma 2.3, we can easily find that if $\mathbb{T}=\mathbb{R}$, then the monotonicity assumption of $p(t)$ can be omitted.

By Lemma 2.3, under the hypothesis

(H1) $p(t)$ is nondecreasing in $[0, \sigma(T)]_{\mathbb{T}}$, and $f(t, 0)>0$ for $t \in[0, T]_{\mathbb{T}}$,

in order to prove the existence of a positive solution of (1.2), it suffices to consider the existence of a solution of (2.1). Now we establish the corresponding variational formulations for (2.1). We set

$$
E=\left\{u \in H_{\Delta}^{1}\left(\left[0, \sigma^{2}(T)\right]\right)_{\mathbb{T}} \mid u(0)=0 \text { if } \alpha_{2}=0, u\left(\sigma^{2}(T)\right)=0 \text { if } \alpha_{4}=0\right\},
$$

then $E$ is a Banach space with the norm $\|\cdot\|$, and we can find that $\|\cdot\|_{0}$ can be taken as an equivalent norm on $E$. Define the functional $I$ on $E$ as

$$
\begin{aligned}
I(u)= & \frac{1}{2} \int_{0}^{\sigma^{2}(T)} p(t)\left|u^{\Delta}(t)\right|^{2} \Delta t+\frac{1}{2} \int_{0}^{\sigma(T)} q(t)\left|u^{\sigma}(t)\right|^{2} \Delta t-\int_{0}^{\sigma(T)} F\left(t,\left(u^{+}\right)^{\sigma}(t)\right) \Delta t \\
& -\int_{0}^{\sigma(T)} f(t, 0)\left(u^{-}\right)^{\sigma}(t) \Delta t+\frac{1}{2} \beta_{1} p(0) u^{2}(0)+\frac{1}{2} \beta_{2} p(\sigma(T)) u^{2}\left(\sigma^{2}(T)\right),
\end{aligned}
$$

where $F(t, \xi)=\int_{0}^{\xi} f(t, s) d s$.

Note that the appearance of the term $\int_{0}^{\sigma(T)} f(t, 0)\left(u^{-}\right)^{\sigma}(t) \Delta t$ in the functional $I$ guarantees that $I$ is $C^{1}$, see next lemma. By the definition of Fréchet derivative and the fact that the immersion $H_{\Delta}^{1}\left(\left[0, \sigma^{2}(T)\right]_{\mathbb{T}}\right) \hookrightarrow C\left(\left[0, \sigma^{2}(T)\right]_{\mathbb{T}}\right)$ is compact, we have the following results.

Lemma 2.5 The following statements are valid. 
(i) $I \in C^{1}(E, \mathbb{R})$, and for every $u, v \in E$,

$$
I^{\prime}(u) v=(u, v)_{0}-\int_{0}^{\sigma(T)} f\left(t,\left(u^{+}\right)^{\sigma}(t)\right) v^{\sigma}(t) \Delta t
$$

(ii) We define

$$
J(u)=\int_{0}^{\sigma(T)} F\left(t,\left(u^{+}\right)^{\sigma}(t)\right) \Delta t+\int_{0}^{\sigma(T)} f(t, 0)\left(u^{-}\right)^{\sigma}(t) \Delta t, \quad u \in E .
$$

Then

$$
J^{\prime}(u) v=\int_{0}^{\sigma(T)} f\left(t,\left(u^{+}\right)^{\sigma}(t)\right) v^{\sigma}(t) \Delta t, \quad u, v \in E
$$

$J$ is weakly continuous in $E$ and $J^{\prime}$ is compact.

(iii) The solutions of (2.1) match up to the critical points of I in E.

For the eigenvalue problem

$$
\left\{\begin{array}{l}
-\left(p(t) u^{\Delta}(t)\right)^{\Delta}+q(t) u^{\sigma}(t)=\lambda u^{\sigma}(t), \quad t \in[0, T]_{\mathbb{T}}, \\
\alpha_{1} u(0)-\alpha_{2} u^{\Delta}(0)=0, \quad \alpha_{3} u\left(\sigma^{2}(T)\right)+\alpha_{4} u^{\Delta}(\sigma(T))=0,
\end{array}\right.
$$

we have the following lemma.

Lemma 2.6 [14, Lemma 3.1] The eigenvalues of (2.6) may be arranged as $0<\lambda_{1}<\lambda_{2}<\cdots$, and there exists a countable orthonormal basis of $E$ consisting of eigenfunction associated eigenvalues of (2.6) and

$$
\lambda_{1}=\inf _{u \in E, u \neq 0} \frac{\|u\|_{0}^{2}}{\int_{0}^{\sigma(T)}\left|u^{\sigma}(t)\right|^{2} \Delta t} .
$$

Remark 2.7 By (2.7) and Lemma 2.2, we know the eigenfunction $\varphi_{1}(t)$ corresponding to the eigenvalue $\lambda_{1}$ satisfies $\varphi_{1}(t)>0$ for $t \in\left(0, \sigma^{2}(T)\right)_{\mathbb{T}}$. Furthermore, by the Krein-Rutman theorem [19, Theorem 7.C], we know $\varphi_{1} \in K$ with

$$
\begin{aligned}
K= & \left\{u \in E \mid u(t)>0 \text { for } t \in\left(0, \sigma^{2}(T)\right)_{\mathbb{T}}, u^{\Delta}(0)>0 \text { if } \alpha_{1}>0, u(0)>0 \text { if } \alpha_{1}=0,\right. \\
& \left.u^{\Delta}(\sigma(T))<0 \text { if } \alpha_{3}>0, u\left(\sigma^{2}(T)\right)>0 \text { if } \alpha_{3}=0\right\} .
\end{aligned}
$$

Lemma 2.8 [1, Theorem 4.73], [8] The problem (1.2) has the Green function

$$
G_{q}(t, s)= \begin{cases}\frac{1}{w} \varphi(t) \psi(\sigma(s)), & t \leq s \\ \frac{1}{w} \varphi(\sigma(s)) \psi(t), & t \geq \sigma(s)\end{cases}
$$

where $w=p(t)\left[\varphi^{\Delta}(t) \psi(t)-\varphi(t) \psi^{\Delta}(t)\right]=$ const $>0, \psi, \varphi$ are solutions of

$$
-\left(p(t) u^{\Delta}(t)\right)^{\Delta}+q(t) u^{\sigma}(t)=0 \quad \text { for } t \in[0, T]_{\mathbb{T}}, \quad u(0)=\alpha_{2}, \quad u^{\Delta}(0)=\alpha_{1}
$$


and

$$
\begin{aligned}
& -\left(p(t) u^{\Delta}(t)\right)^{\Delta}+q(t) u^{\sigma}(t)=0 \quad \text { for } t \in[0, T]_{\mathbb{T}} \\
& u\left(\sigma^{2}(T)\right)=\alpha_{4}, \quad u^{\Delta}(\sigma(T))=-\alpha_{3},
\end{aligned}
$$

respectively, and satisfy $\varphi(t)>0, t \in\left(0, \sigma^{2}(T)\right]_{\mathbb{T}}, \varphi^{\Delta}(t) \geq 0, t \in[0, \sigma(T)]_{\mathbb{T}}, \psi(t)>0, t \in$ $\left[0, \sigma^{2}(T)\right)_{\mathbb{T}}, \psi^{\Delta}(t) \leq 0, t \in[0, \sigma(T)]_{\mathbb{T}}$.

\section{Lemma 2.9 The function defined by}

$$
\Gamma(t, s)=\frac{G_{q}(t, s)}{\varphi_{1}^{\sigma}(s)}
$$

belongs to $L^{\infty}\left([0, \sigma(T)]_{\mathbb{T}} \times[0, \sigma(T)]_{\mathbb{T}}\right)$, where $\varphi_{1}$ is given in Remark 2.7 .

Proof Clearly, $\Gamma(t, s)$ is well defined in $[0, \sigma(T)]_{\mathbb{T}} \times(0, \sigma(T))_{\mathbb{T}}$.

If $\alpha_{2}>0$, by $\varphi_{1} \in K$ and $\alpha_{1} \varphi_{1}(0)-\alpha_{2} \varphi_{1}^{\Delta}(0)=0$, we have $\varphi_{1}(0)>0$. Hence $\varphi_{1}(\sigma(0))>0$.

If $\alpha_{2}=0$, then $\varphi_{1}(0)=0$. By Remark 2.7, we know $\varphi_{1}^{\Delta}(0)>0$. If $\sigma(0)>0$, then $u^{\sigma}(0)>0$. If $\sigma(0)=0$, then there exists $\delta>0$ such that $\varphi_{1}(t)>0, \varphi_{1}^{\Delta}(t)>0$ for $t \in(0, \delta)_{\mathbb{T}}$, then by L'Hôspital rule [1, Theorem 1.119] and Lemma 2.8, we know

$$
\begin{aligned}
-\infty & <\lim _{\delta \rightarrow 0^{+}} \inf _{s \in(0, \delta)_{\mathbb{T}}} \frac{\varphi^{\Delta}(s)}{\varphi_{1}^{\Delta}(s)} \leq \lim _{\delta \rightarrow 0^{+}} \inf _{s \in(0, \delta)_{\mathbb{T}}} \frac{\varphi(s)}{\varphi_{1}(s)} \\
& \leq \lim _{\delta \rightarrow 0^{+}} \sup _{s \in(0, \delta)_{\mathbb{T}}} \frac{\varphi(s)}{\varphi_{1}(s)} \leq \lim _{\delta \rightarrow 0^{+}} \sup _{s \in(0, \delta)_{\mathbb{T}}} \frac{\varphi^{\Delta}(s)}{\varphi_{1}^{\Delta}(s)}<+\infty .
\end{aligned}
$$

Hence, $\Gamma(t, s)$ is bounded for $s$ close to 0 .

Similarly, we can derive that $\Gamma(t, s)$ is bounded for $s$ close to $\sigma(T)$.

In order to derive the main result, we list the following well-known mountain pass lemma.

Lemma 2.10 [20, Theorem 6.1] Suppose $I \in C^{1}(E, R)$ satisfies the P.S. condition. Suppose $I(0)=0$ and

(i) there exist $\rho>0, \alpha>0$ such that $I(u) \geq \alpha$ for $u \in E$ with $\|u\|=\rho$;

(ii) there is $u_{1} \in E$ such that $\left\|u_{1}\right\|>\rho$ and $I\left(u_{1}\right)<\alpha$.

Define

$$
P=\left\{p \in C^{0}([0,1] ; E) ; p(0)=0, p(1)=u_{1}\right\}
$$

Then $\beta=\inf _{p \in P} \sup _{u \in p} I(u) \geq \alpha$ is a critical value.

\section{Main results}

In this section, we establish some existence criteria of a positive solution of (1.1) by employing the sub-supersolution method and critical point theory.

First, using a method analogous to that in [21], we construct a supersolution to employ the sub-supersolution method. 
Theorem 3.1 Assume that (H1) holds and there are constants $a_{1}>0$ and $a_{2} \geq a_{1} a_{\mu}$ such that

$$
f(t, \xi)<\mu \xi+a_{1}, \quad t \in[0, T]_{\mathbb{T}}, \xi \in\left[0, a_{2}\right]
$$

where $\mu<\lambda_{1}$ is fixed, $a_{\mu}=\left\|u_{\mu}\right\|_{\infty}, u_{\mu}$ represents the unique positive solution of

$$
\left\{\begin{array}{l}
-\left(p(t) u^{\Delta}(t)\right)^{\Delta}+q(t) u^{\sigma}(t)=\mu u^{\sigma}(t)+1, \quad t \in[0, T]_{\mathbb{T}}, \\
\alpha_{1} u(0)-\alpha_{2} u^{\Delta}(0)=0, \quad \alpha_{3} u\left(\sigma^{2}(T)\right)+\alpha_{4} u^{\Delta}(\sigma(T))=0 .
\end{array}\right.
$$

Then the problem (1.2) has at least one positive solution.

Proof For fixed $\mu<\lambda_{1}$, let $v$ be the unique positive solution of

$$
\left\{\begin{array}{l}
-\left(p(t) u^{\Delta}(t)\right)^{\Delta}+q(t) u^{\sigma}(t)=\mu u^{\sigma}(t)+a_{1}, \quad t \in[0, T]_{\mathbb{T}}, \\
\alpha_{1} u(0)-\alpha_{2} u^{\Delta}(0)=0, \quad \alpha_{3} u\left(\sigma^{2}(T)\right)+\alpha_{4} u^{\Delta}(\sigma(T))=0 .
\end{array}\right.
$$

Then, from the definition of $u_{\mu}$, we have $\|v\|_{\infty} \leq a_{1} a_{\mu}$. Then, by the assumptions, it is easy to see that $v$ is a supersolution of (1.2). In addition, condition (H1) guarantees that the constant function 0 is a strict subsolution of (1.2). Therefore, the sub-supersolution method implies (1.2) has a positive solution $u_{1}$.

Remark 3.2 Furthermore, by Lemma 2.8, we know

$$
a_{\mu} \leq \int_{0}^{\sigma(T)} \max _{t \in[0, T]_{\mathbb{T}}}\left|G_{q-\mu}(t, s)\right| \Delta s .
$$

Theorem 3.3 Under the hypothesis of Theorem 3.1 and suppose the condition

(H2) $\liminf _{\xi \rightarrow+\infty} \frac{f(t, \xi)}{\xi}>\lambda_{1}$ uniformly for $t \in[0, T]_{\mathbb{T}}$

holds, then the problem (1.2) has at least two positive solutions.

In order to prove this theorem, we first present some necessary lemmas.

Lemma 3.4 Let $v, u_{1}$ be given in the proof of Theorem 3.1, then $u_{1}$ is a local minimizer of I in $E$.

Proof Denote

$$
W=\left\{u \in C^{1}\left([0, \sigma(T)]_{\mathbb{T}}, \mathbb{R}\right) \mid \alpha_{1} u(0)-\alpha_{2} u^{\Delta}(0)=0, \alpha_{3} u\left(\sigma^{2}(T)\right)+\alpha_{4} u^{\Delta}(\sigma(T))=0\right\} .
$$

By the assumptions and Lemma 2.8, we easily find $v-u_{1} \in K, u_{1} \in K$. Hence $u_{1}$ is a local minimizer of $I$ in $W$.

Next, by a similar argument to that in [22], we assert that $u_{1}$ is also a local minimizer of $I$ in $E$.

In fact, if $u_{1}$ is not a local minimizer of $I$ in $E$, then for every $\varepsilon>0$ there is $v_{\varepsilon} \in E$ such that $0<\left\|v_{\varepsilon}\right\| \leq \varepsilon, I\left(u_{1}+v_{\varepsilon}\right)<I\left(u_{1}\right)$ and $I\left(u_{1}+v_{\varepsilon}\right)=\inf _{v \in E,\|\nu\| \leq \varepsilon} I\left(v+u_{1}\right)$. By the Lagrange multiplier rule, we know there exists a constant $\mu_{\varepsilon} \leq 0$ such that

$$
I^{\prime}\left(u_{1}+v_{\varepsilon}\right) \varphi=\mu_{\varepsilon}\left(v_{\varepsilon}, \varphi\right)_{0} \quad \text { for every } \varphi \in E .
$$


Note that $u_{1}$ is a solution of (1.2), so

$$
\begin{aligned}
I^{\prime}\left(u_{1}+v_{\varepsilon}\right) \varphi & =\left(u_{1}+v_{\varepsilon}, \varphi\right)_{0}-\int_{0}^{\sigma(T)} f\left(t, u_{1}^{\sigma}+v_{\varepsilon}^{\sigma}\right) \varphi^{\sigma} \Delta t \\
& =\left(v_{\varepsilon}, \varphi\right)_{0}-\int_{0}^{\sigma(T)} f\left(t, u_{1}^{\sigma}+v_{\varepsilon}^{\sigma}\right) \varphi^{\sigma} \Delta t+\int_{0}^{\sigma(T)} f\left(t, u_{1}^{\sigma}\right) \varphi^{\sigma} \Delta t .
\end{aligned}
$$

Thus, from (3.2), we have

$$
\left(1-\mu_{\varepsilon}\right)\left(v_{\varepsilon}, \varphi\right)_{0}-\int_{0}^{\sigma(T)} f\left(t, u_{1}^{\sigma}+v_{\varepsilon}^{\sigma}\right) \varphi^{\sigma} \Delta t+\int_{0}^{\sigma(T)} f\left(t, u_{1}^{\sigma}\right) \varphi^{\sigma} \Delta t=0 .
$$

Therefore, $v_{\varepsilon}$ is a solution of

$$
\left\{\begin{array}{l}
-\left(1-\mu_{\varepsilon}\right)\left(p(t) u^{\Delta}(t)\right)^{\Delta}+q(t) u^{\sigma}(t) \\
\quad=f\left(t, u^{\sigma}(t)+u_{1}^{\sigma}(t)\right)-f\left(t, u_{1}^{\sigma}(t)\right), \quad t \in[0, T]_{\mathbb{T}}, \\
\alpha_{1} u(0)-\alpha_{2} u^{\Delta}(0)=0, \quad \alpha_{3} u\left(\sigma^{2}(T)\right)+\alpha_{4} u^{\Delta}(\sigma(T))=0 .
\end{array}\right.
$$

It is easy to show that $v_{\varepsilon} \rightarrow 0$ as $\varepsilon \rightarrow 0$ in $C^{1}\left([0, \sigma(T)]_{\mathbb{T}}\right)$. But this contradicts the fact that $u_{1}$ is a local minimizer of $I$ in $W$.

Next, under hypothesis (H2), in order to show the existence of the second positive solution of (1.2) by employing the mountain pass lemma, we need to show that $I$ satisfies the P.S. condition. However, by (H2), we cannot justify this; therefore, we consider the truncation function $f_{n}$ and the truncation functional $I_{n}$ defined as follows.

Let $\gamma>\lambda_{1}$ and $\left\{\xi_{n}\right\}$ be an increasing positive sequence with $\xi_{n} \rightarrow+\infty$ as $n \rightarrow+\infty$. For $n=1,2, \ldots$, define

$$
f_{n}(t, \xi)= \begin{cases}f(t, 0), & \xi<0, \\ f(t, \xi), & 0 \leq \xi \leq \xi_{n}, \\ \gamma\left(\xi-\xi_{n}\right)+f\left(t, \xi_{n}\right), & \xi>\xi_{n}\end{cases}
$$

and

$$
I_{n}(u)=\frac{1}{2}\|u\|_{0}^{2}-\int_{0}^{\sigma(T)} F_{n}\left(t,\left(u^{+}\right)^{\sigma}(t)\right) \Delta t-\int_{0}^{\sigma(T)} f(t, 0)\left(u^{-}\right)^{\sigma}(t) \Delta t, \quad u \in E,
$$

where $F_{n}(t, s)=\int_{0}^{s} f_{n}(t, \xi) d \xi$. Then

$$
I_{n}^{\prime}(u) v=(u, v)_{0}-\int_{0}^{\sigma(T)} f_{n}\left(t,\left(u^{+}\right)^{\sigma}(t)\right) v^{\sigma}(t) \Delta t, \quad u, v \in E .
$$

Lemma 3.5 Assume that (H1) and (H2) hold, then there exists $n_{0}>0$ such that the functional $I_{n}$ satisfies the P.S. condition in $E$ for $n>n_{0}$.

Proof For given $n$, let $\left\{u_{m}\right\} \subseteq E$ be the P.S. sequence of $I_{n}$, that is, $\left\{I_{n}\left(u_{m}\right)\right\}$ is bounded and $I_{n}^{\prime}\left(u_{m}\right) \rightarrow 0$ as $m \rightarrow \infty$. If $\left\{u_{m}\right\}$ is bounded, one can deduce that $I$ satisfies the P.S. condition by a similar proof to Proposition B.35 in [23]. 
Suppose that $\left\{u_{m}\right\}$ is unbounded. Since

$$
\left\|u_{m}^{-}\right\|_{0}^{2} \leq-\int_{0}^{\sigma(T)} f(t, 0)\left(u_{m}^{-}\right)^{\sigma}(t) \Delta t+\left(u_{m}, u_{m}^{-}\right)_{0}=I_{n}^{\prime}\left(u_{m}\right) u_{m}^{-} \leq\left\|I_{n}^{\prime}\left(u_{m}\right)\right\|\left\|u_{m}^{-}\right\|_{0},
$$

we have $\left\|u_{m}^{-}\right\|_{0} \rightarrow 0$ as $m \rightarrow \infty$. Denote $v_{m}=\frac{u_{m}}{\left\|u_{m}\right\|_{0}}$, then $\left\|v_{m}\right\|_{0}=1$. So, without loss of generality, we can assume that $v_{m} \rightarrow v \geq 0$ in $E, v_{m} \rightarrow v$ in $C\left(\left[0, \sigma^{2}(T)\right]_{\mathbb{T}}\right)$. Note that

$$
\frac{I_{n}^{\prime}\left(u_{m}\right)\left(v_{m}-v\right)}{\left\|u_{m}\right\|_{0}}=\left(v_{m}, v_{m}-v\right)_{0}-\frac{1}{\left\|u_{m}\right\|_{0}} \int_{0}^{\sigma(T)} f_{n}\left(t,\left(u_{m}^{+}\right)^{\sigma}(t)\right)\left(v_{m}-v\right)^{\sigma}(t) \Delta t,
$$

by the definition of $f_{n}$ and passing to limit in (3.4), one can derive that $\|v\|_{0}=1$.

In view of $(\mathrm{H} 2)$ and the definition of $f_{n}$, for $\varepsilon>0$ small enough, there exist $\xi^{*}>0$ independent of $n, n_{0}>0$ such that

$$
f_{n}(t, \xi)>\left(\lambda_{1}+\varepsilon\right) \xi \quad \text { for } \xi>\xi^{*}, n>n_{0} .
$$

Since $\left(u_{m}^{\sigma}, \varphi_{1}\right)_{0}=\lambda_{1} \int_{0}^{\sigma(T)} u_{m}^{\sigma}(t) \varphi_{1}^{\sigma}(t) \Delta t$, then

$$
\begin{aligned}
\varepsilon \int_{0}^{\sigma(T)} v_{m}^{\sigma}(t) \varphi_{1}^{\sigma}(t) \Delta t \\
=\frac{1}{\left\|u_{m}\right\|_{0}}\left(\int_{0}^{\sigma(T)}\left(\lambda_{1}+\varepsilon\right) u_{m}^{\sigma}(t) \varphi_{1}^{\sigma}(t) \Delta t-\left(u_{m}, \varphi_{1}\right)_{0}\right) \\
=\frac{1}{\left\|u_{m}\right\|_{0}}\left(\int_{0}^{\sigma(T)}-f_{n}\left(t,\left(u_{m}^{+}\right)^{\sigma}(t)\right) \varphi_{1}^{\sigma}(t)+\left(\lambda_{1}+\varepsilon\right)\left(u_{m}^{+}\right)^{\sigma}(t) \varphi_{1}^{\sigma}(t) \Delta t-I_{n}^{\prime}\left(u_{m}\right) \varphi_{1}\right. \\
\left.\quad+\int_{0}^{\sigma(T)}\left(\lambda_{1}+\varepsilon\right)\left(u_{m}^{-}\right)^{\sigma}(t) \varphi_{1}^{\sigma}(t) \Delta t\right) .
\end{aligned}
$$

Passing to the limit in (3.6), we know $\varepsilon \int_{0}^{\sigma(T)} v^{\sigma}(t) \varphi_{1}^{\sigma}(t) \Delta t \leq 0$. Hence $v \equiv 0$, which contradicts $\|v\|_{0}=1$. Therefore $\left\{u_{m}\right\}$ is bounded. So, $I_{n}$ satisfies the P.S. condition for $n>n_{0}$.

By Lemmas 3.4 and 3.5, we deduce that for $n$ large enough, $I_{n}$ has a nontrivial critical point $w_{n}$ by using the mountain pass lemma and Theorem 1 in [24]. In order to obtain a solution of (1.2), we need to get an estimate of $w_{n}$. Therefore, we first give an estimate of a nonnegative solution of (1.2) employing a method similar to that in [25].

Lemma 3.6 Suppose (H2) holds, then there is $M>0$ such that for any nonnegative solution $u$ of (1.2), we have $\|u\|_{\infty} \leq M$.

Proof If $u$ is a nonnegative solution of (1.2), then by the definition of $\varphi_{1}$, we have

$$
\begin{aligned}
\lambda_{1} \int_{0}^{\sigma(T)} u^{\sigma}(t) \varphi_{1}^{\sigma}(t) \Delta t= & \int_{0}^{\sigma^{2}(T)} p(t) u^{\Delta}(t) \varphi_{1}^{\Delta}(t) \Delta t+\int_{0}^{\sigma(T)} q(t) u^{\sigma}(t) \varphi_{1}^{\sigma}(t) \Delta t \\
& +\frac{1}{2} \beta_{1} p(0) u(0) \varphi_{1}(0)+\frac{1}{2} \beta_{2} p(\sigma(T)) u\left(\sigma^{2}(T)\right) \varphi_{1}\left(\sigma^{2}(T)\right) \\
& =\int_{0}^{\sigma(T)} f\left(t, u^{\sigma}(t)\right) \varphi_{1}^{\sigma}(t) \Delta t .
\end{aligned}
$$


Condition (H2) implies there exist $\rho>\lambda_{1}, C>0$ such that

$$
f(t, \xi) \geq \rho \xi-C, \quad t \in[0, T], \xi \geq 0 .
$$

Hence, from (3.7) and (3.8), we derive that

$$
\int_{0}^{\sigma(T)} u^{\sigma}(t) \varphi_{1}^{\sigma}(t) \Delta t \leq \frac{C}{\rho-\lambda_{1}} \int_{0}^{\sigma(T)} \varphi_{1}^{\sigma}(t) \Delta t
$$

So, using (3.7), we know

$$
\int_{0}^{\sigma(T)} f\left(t, u^{\sigma}(t)\right) \varphi_{1}^{\sigma}(t) \Delta t \leq \frac{\lambda_{1} C}{\rho-\lambda_{1}} \int_{0}^{\sigma(T)} \varphi_{1}^{\sigma}(t) \Delta t
$$

Thus, by (H2), we know $\int_{0}^{\sigma(T)}\left|f\left(t, u^{\sigma}(t)\right)\right| \varphi_{1}^{\sigma}(t) \Delta t$ is uniformly bounded. Note that by Lemma 2.9, for $t \in\left[0, \sigma^{2}(T)\right]$,

$$
|u(t)|=\left|\int_{0}^{\sigma(T)} G_{q}(t, s) f\left(s, u^{\sigma}(s)\right) \Delta s\right| \leq\|\Gamma\|_{\infty} \int_{0}^{\sigma(T)}\left|f\left(s, u^{\sigma}(s)\right)\right| \varphi_{1}^{\sigma}(s) \Delta s .
$$

Hence, there exists $M>0$ such that $\|u\|_{\infty} \leq M$.

Remark 3.7 Note that we only need (H2) to derive (3.8). Hence, (3.5) implies Lemma 3.6 is also valid for the truncation problem.

Proof of Theorem 3.3 Since the positive solution $u_{1}$ derived from Theorem 3.1 is a local minimizer of $I$ and $0<u_{1} \leq v$, we can choose $n_{0}$ large enough such that $I\left(u_{1}\right)=I_{n}\left(u_{1}\right)$ for every $n>n_{0}$. Hence, $u_{1}$ is also a local minimizer of $I_{n}$ for $n>n_{0}$. Then, from the definition of $f_{n}$ and Lemma 3.5, we know the mountain pass lemma and Theorem 1 in [24] imply that $I_{n}$ has the second critical point $u_{2}$. Furthermore, by Remark 3.7, we know $u_{2}$ is also a critical point of $I$. Thus the problem (1.2) has the second positive solution $u_{2}$.

\section{Competing interests}

The authors declare that they have no competing interests.

\section{Authors' contributions}

All the authors typed, read, and approved the final manuscript.

\section{Author details}

'School of Mathematics and Statistics, Lanzhou University, Lanzhou, Gansu 730000, People's Republic of China. ${ }^{2}$ Center of Teaching Guidance, Gansu Radio and TV University, Lanzhou, Gansu 730000, People's Republic of China.

\section{Acknowledgements}

Dedicated to Professor Hari M Srivastava.

The research of HR Sun has been supported by the program for New Century Excellent Talents in University (NECT-12-0246) and FRFCU (Izujbky-2013-k02).

Received: 20 January 2013 Accepted: 26 April 2013 Published: 13 May 2013

\section{References}

1. Bohner, M, Peterson, A: Dynamic Equations on Time Scales. Birkhäuser, Boston (2001)

2. Bohner, M, Peterson, A: Advances in Dynamic Equations on Time Scales. Birkhäuser, Boston (2003)

3. Hilger, S: Ein Maßkettenkalkül mit Anwendung auf Zentrumsmannigfaltigkeiten. Ph.D. thesis, Universität Würzburg (1988) (in German) 
4. Agarwal, RP, Otero-Espinar, V, Perera, K, Vivero, DR: Existence of multiple positive solutions for second order nonlinear dynamic BVPs by variational methods. J. Math. Anal. Appl. 331, 1263-1274 (2007)

5. Agarwal, RP, Otero-Espinar, V, Perera, K, Vivero, DR: Multiple positive solutions of singular Dirichlet problems on time scales via variational methods. Nonlinear Anal. 67, 368-381 (2007)

6. Anderson, D: Solutions to second-order three-points problems on time scales. J. Differ. Equ. Appl. 8, 673-688 (2002)

7. Atici, EM, Guseinov, GS: On Green's functions and positive solutions for boundary value problems on time scales. J. Comput. Appl. Math. 141, 75-99 (2002)

8. Erbe, L, Peterson, A, Mathsen, R: Existence, multiplicity, and nonexistence of positive solutions to a differential equation on a measure chain. J. Comput. Appl. Math. 113, 365-380 (2000)

9. Rynne, BP: $L^{2}$ spaces and boundary value problems on time scales. J. Math. Anal. Appl. 328, 1217-1236 (2007)

10. Sun, HR: Triple positive solutions for $p$-Laplacian $m$-point boundary value problem on time scales. Comput. Math. Appl. 58, 1736-1741 (2009)

11. Sun, HR, Li, WT: Positive solutions for nonlinear three-point boundary value problems on time scales. J. Math. Anal. Appl. 299, 508-524 (2004)

12. Sun, HR, Li, WT: Existence theory for positive solutions to one-dimensional $p$-Laplacian boundary value problems on time scales. J. Differ. Equ. 240, 217-248 (2007)

13. Jiang, L, Zhou, Z: Existence of weak solutions of two-point boundary value problems for second-order dynamic equations on time scales. Nonlinear Anal. 69, 1376-1388 (2008)

14. Zhang, QG, Sun, HR: Variational approach for Sturm-Liouville boundary value problems on time scales. J. Appl. Math. Comput. 36, 219-232 (2011)

15. Bonanno, G, Candito, P: Non-differentiable functionals and applications to elliptic problems with discontinuous nonlinearities. J. Differ. Equ. 244, 3031-3059 (2008)

16. Agarwal, RP, Otero-Espinar, V, Perera, K, Vivero, DR: Basic properties of Sobolev's spaces on time scales. Adv. Differ. Equ. 2006, Article ID 38121 (2006)

17. Guseinov, GS: Integration on time scales. J. Math. Anal. Appl. 285, 107-127 (2003)

18. Cabada, A, Vivero, DR: Criterions for absolute continuity on time scales. J. Differ. Equ. Appl. 11, 1013-1028 (2005)

19. Zeidler, E: Nonlinear Functional Analysis and Its Application I: Fixed-Point Theorems. Springer, New York (1985)

20. Struwe, M: Variational Methods. Springer, Berlin (1990)

21. DE Figueiredo, DG, Lions, PL: On pairs of positive solutions for a class of semilinear elliptic problems. Indiana Univ. Math. J. 34, 591-606 (1985)

22. Brezis, H, Nirenberg, L: $H^{1}$ versus $C^{1}$ local minimizers. C. R. Acad. Sci., Sér. 1 Math. 317, 465-472 (1993)

23. Rabinowitz, PH: Minimax Methods in Critical Point Theory with Applications to Differential Equations. CBMS Reg. Conf. Ser. Math., vol. 65. Am. Math. Soc., Providence (1986)

24. Ghoussoub, N, Preiss, D: A general mountain pass principle for locating and classifying critical points. Ann. Inst. Henri Poincaré, Anal. Non Linéaire 6, 321-330 (1989)

25. Mawhin, J, Omana, W: A priori bounds and existence of positive solutions for some Sturm-Liouville superlinear boundary value problems. Funkc. Ekvacioj 35, 333-342 (1992)

doi:10.1186/1687-2770-2013-123

Cite this article as: Zhang et al.: Positive solutions for Sturm-Liouville BVPs on time scales via sub-supersolution and variational methods. Boundary Value Problems 2013 2013:123.

\section{Submit your manuscript to a SpringerOpen ${ }^{\circ}$ journal and benefit from:}

- Convenient online submission

Rigorous peer review

- Immediate publication on acceptance

Open access: articles freely available online

- High visibility within the field

- Retaining the copyright to your article 